\title{
MPEG-SCORM: ontologia de metadados interoperáveis entre TV Digital e e-Learning
}

\author{
M. Correia Santos, Y. Iano, Member, IEEE
}

\begin{abstract}
The convergence of digital media offers an integration of ICT focused on telecommunications and multimedia domain (under responsibility of the Moving Picture Experts Group, ISO/IEC JTC1 SC29) and the ICTE (the ICT for Education), managed by the ISO/IEC JTC1 SC36, highlighting the MPEG standards, employees as content and metadata to the multimedia Digital TV and the technologies applied to e-Learning. Regarding this, there is the problem of developing an interoperable matching for normative bases, achieving an innovative proposal in the convergence between digital telecommunications and applications for e-Learning, also essentially multimedia. To reach this purpose it is proposed to create a standard ontology of interoperable metadata for web, Digital TV and extensions for mobile devices based on the integration between MPEG-21 and SCORM metadata standards. The methodology used consists on building ontology MPEG-21 SCORM which can be achieved on making a correspondence through the XPath language, managed by the W3C. The employ of the XPath language is desirable for matching and mapping both metadata schema patterns integrating MPEG-21 (mostly Digital Item Declaration Language) and SCORM metadata schema. The practical purpose is the creation and storage of objects for use in digital telecommunications as Digital Television, in an interoperable way with the e-Learning industry, here as description metadata for all sorts of media and hypermedia to create learning objects.

In the First Chapter, there will be presented the Digital Television system based on MPEG video and its scenario. Chapter II will discuss about the concept of Learning Objects and Metadata for e-Learning standards, highlighting SCORM. Chapter III covers the issue of MPEG-21 metadata specifically, focusing on its structure and normalization as well. Finally, Chapter IV presents the adopted methodology for integration and interoperability between multimedia and e-learning metadata standards, based on MPEG-21 and SCORM through the W3C XPath mapping language.
\end{abstract}

Index Terms - Digital Television, e-Learning, Metadata, MPEG, SCORM.

\section{INTRODUÇÃO}

A inovação tecnológica em questão compreende a pesquisa de um novo padrão que integra as normalizações internacionais implementadas na área do multimídia, destacadamente a Televisão Digital interativa (pelo SC29 ${ }^{1}$ ), e, do outro lado, com as normalizações advindas do domínio da Educação e suas tecnologias $\left(\mathrm{SC} 36^{2}\right)$.

Trabalho submetido ao Congresso SET 2015 em 27/5/2015. Este trabalho recebeu o apoio financeiro parcial da CAPES - Brasil. Marcelo Correia Santos é Doutorando do Programa de Engenharia Elétrica da
No contexto brasileiro, a Televisão Digital, sob a ótica do SBTVD $^{3}$, foi concebida com o propósito de cumprir algumas missões como contrapartida à sociedade brasileira, entre elas levar inclusão digital e educação à população, em todas as regiões do país, vindo de encontro a uma demanda latente.

A Educação a Distância, ou o e-Learning, em seu formato para Televisão chamado T-learning ${ }^{4}$, é ferramenta importante para o cumprimento dessas metas, agregando à cadeia de valor de TV e conferindo desenvolvimento ao país.

Como uma Nova Tecnologia Digital da Informação e Comunicação, a Televisão Digital assume seu lugar como mídia expoente no processo de convergência digital, visto que, entre outros atributos, possibilita a coexistência de recursos hipermidiáticos convergentes sobre a plataforma digital.

A inovação tecnológica em questão compreende a pesquisa de um novo padrão que integra as normalizações internacionais implementadas na área do multimídia, destacadamente a Televisão Digital interativa (pelo SC29), e, do outro lado, com as normalizações advindas do domínio da Educação e suas tecnologias (SC36).

Tecnicamente, a Televisão Digital faz parte do domínio do multimídia $\mathrm{MPEG}^{5}$, pelo fato da TV Digital já empregar como formato de exibição, atualmente, o padrão de vídeo digital MPEG-4 AVC H264, consolidado como o padrão da indústria atualmente.

Entretanto, a padronização do universo do multimídia MPEG não se restringe ao MPEG-4, assim como não se restringiu ao já igualmente bem sucedido MPEG-2.

Há uma década e meia fazem-se grandes projetos de pesquisa, experiências e implementações práticas em aplicações com excelente desempenho que se utilizam dos

UNICAMP (avaliação 7 CAPES) e atualmente Professor do Centro Universitário SENAC-SP.

Yuzo Iano, membro do IEEE, é Prof. Dr. do Depto. de Telecomunicações e Telemática da FEEC UNICAMP e Editor Chefe do SET International Journal of Broadcast Engineering.

${ }^{1}$ ISO/IEC JTC1 SC29, comitê que trata da codificação de som, de imagem e da informação multimídia e hipermídia

${ }^{2}$ ISO/IEC JTC1 SC36, comitê que trata das tecnologias para a educação, informação e a aprendizagem

3 A sigla SBTVD refere-se a Sistema Brasileiro de Televisão Digital. Disponível em: $<$ http://sbtvd.cpqd.com.br $>$. Acesso em: 10 fev. 2015.

${ }^{4}$ E-Learning (aprendizado eletrônico) via mídia Televisão Digital.

${ }^{5}$ Moving Picture Experts, grupo de trabalho da ISO/IEC estabelecido em 1988, com a missão de desenvolver padrões para representação codificada de dados relacionados em áudio e vídeo digital 
padrões MPEG-7 e MPEG-21, além de outros consolidados, como o TV-Anytime ${ }^{6}$.

Após a padronização dos formatos MPEG-1 e MPEG-2, duas normas que tornaram possíveis o multimídia e o vídeo DVD, a partir do advento da alta definição e, por conseguinte, da Televisão Digital, o MPEG-4, 7 e 21 tornaram-se as mais ativas e importantes normas na pauta do SC29. Estas três normas articulam-se de forma interdependente entre si, prolongam-se ou se complementam de maneira coerente.

Estas diferentes normas MPEG e suas características em termos de qualidade de codificação, de eficácia de compressão e de interatividade são sintetizadas na Figura 1:

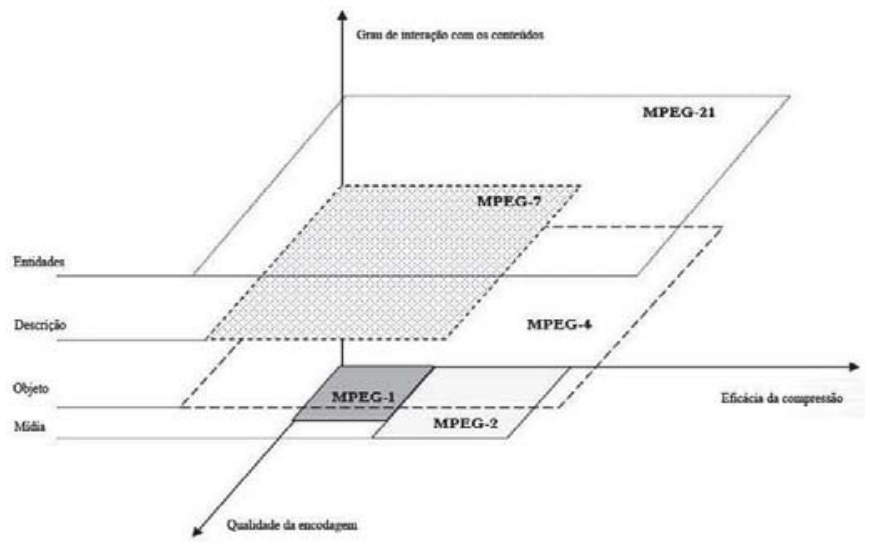

Fig. 1. Diferentes normas MPEG e suas características em termos de qualidade de encodagem, de eficácia de compressão e de interatividade [1] tradução livre.

Considerando-se os desafios de padronização das Tecnologias da Informação e Comunicação aplicadas à Educação, constitui-se um problema de engenharia de Televisão Digital ainda em desenvolvimento a questão de como a família MPEG Vídeo, por meio das normas MPEG-4, 7 e, em especial, MPEG-21 [2], poderia oferecer uma base normativa para a implementação de metadados audiovisuais associados às TICE.

Esse desenvolvimento necessita de uma instrumentalização de todos os recursos ou objetos pedagógicos (além do próprio vídeo, imagem estática, som, texto, hiperlink etc) e deve se situar dentro de um quadro normativo a fim de garantir a interoperabilidade, reutilização e referenciamento pela maioria das plataformas ou ambientes virtuais de aprendizado, ou mesmo pela TV Digital ou outras mídias que venham a se localizar numa dimensão além desses ambientes clássicos educacionais.

A lógica referencial do corpus do documento digital é uma das premissas basais para os desenvolvedores de sistemas de

${ }^{6}$ Especificação de entrega de conteúdo multimídia e seus metadados para TV Digital. Disponível em: <http://www.tv-anytime.org>. Acesso: 10 abr. 2015. informação para que seja possível criar uma normalização no tocante à engenharia de software (XML, MPEG-7, MPEG-21). Esses princípios são desenvolvidos em todas as dimensões pelo subcomitê que trata da padronização para as TICE, o SC36.

Por outro lado, os especialistas da $\mathrm{ADL}^{7}$, que desenvolveram o padrão SCORM, participam do SC36 e de certa forma propõem explorar o MPEG-21 para solucionar uma família de funcionalidades normativas que se deve desenvolver para tornar as normas das TICE viáveis técnica e economicamente.

A ADL, desenvolvedora do padrão SCORM, intervém como parceiro nível A no SC36 - atuando no subcomitê em questão, e sua estratégia é capitalizar sobre as normas de outros SC, e em particular procura atualmente fomentar a adoção da Parte 5 do MPEG-21 [3] (REL, ou Rights Expression Language), para solucionar problemas de copyright, além de delegar às normas do padrão $\mathrm{LOM}^{8}$ (ou outros formatos de metadados de aprendizagem como o Dublin Core ou o futuro MLR) o cuidado de descrever os recursos de aprendizagem em suas diferentes facetas. Claramente, esta descrição se tornaria concorrente das questões das normas multimídia da família MPEG.

\section{OBjetos de Aprendizagem e PADRÕEs DE Metadados}

A definição de Objeto de Aprendizagem (OA), na perspectiva da engenharia, segundo o padrão 1484.12.1 (Standard for Learning Object Metadata) do IEEE [4], seria "um objeto de aprendizagem é definido como qualquer entidade, digital ou não digital, que pode ser utilizada para aprendizado, educação ou treinamento".

No universo digital, esta entidade pode, portanto, ser reutilizada ou referenciada durante o aprendizado apoiado por computador. O mesmo pode conter simples elementos como um texto ou um vídeo. Ou ainda, ele pode ser um hipertexto, um curso ou até mesmo uma animação com áudio e interação.

Segundo a IEEE LTSC [4], o padrão LOM focaliza o mínimo de atributos necessários para permitir que um Objeto de Aprendizagem seja gerenciado, localizado e avaliado. Os metadados permitem a catalogação e a codificação do objeto de Aprendizagem, tornando-o compreensível para as diversas plataformas.

SCORM não define um modelo de metadados: ele reconhece e recomenda enfaticamente o LOM como o padrão de facto [5]. Todavia, SCORM define o XML como sintaxe de representação dos metadados (processo chamado de XML binding). Por isto, ao representarem-se os metadados SCORM, usa-se LOM.

No modelo CAM publicado pela ADL [5], SCORM definiu, em sua parte relativa a Metadados, nove categorias para descrever atributos de objetos de aprendizagem. A diretriz é oferecida para aplicação dos metadados em assets (arquivos de

\footnotetext{
${ }^{7}$ Advanced Distributed Learning, iniciativa do governo americano datada de 1999, tendo como vetor o Departamento de Defesa, que tem a missão de recomendar e desenvolver padrões para software de treinamento.

${ }^{8}$ Learning Object Metadata, padrão de metadados ente os pioneiros, cujo metadata schema é adotado pelo padrão SCORM.
} 
mídia), SCOs (conjuntos de assets), atividades, organizações do conteúdo e agregações de conteúdo, para descrevê-los de uma forma consistente de modo a que possam ser identificados, categorizados, consultados e encontrados nos sistemas, a fim de facilitar o compartilhamento e reutilização. Já a parte que trata do Empacotamento de dados é um conjunto de requisitos e diretrizes específicas; um pacote representa objeto de aprendizagem e é armazenado em repositórios.

É possível realizar-se correspondências entre padrões de metadados, pois não são incompatíveis. Por exemplo, mapeando-se os metadados para e-learning DCMI e SCORM (normalizando com a linguagem XPath), teremos descrita a correspondência na Tabela 1 .

Conforme se observa na Tabela 1, estes identificadores vão desde os mais gerais, até os relativos a direitos autorais (o termo Rights, observável na penúltima linha da tabela), que corresponde como já abordado a um foco da ADL para integração de uma parte da norma MPEG-21 (Parte 5, relativa à linguagem de expressão de direitos) ao padrão SCORM.

TABELA I.

MAPEAMENTO ENTRE METADADOS SCORM (LOM) E DCMI

\begin{tabular}{|c|c|}
\hline \multicolumn{2}{|c|}{ Correspondência entre Metadados SCORM (LOM) / DCMI } \\
\hline SCORM & DCMI \\
\hline /lom/general/identifier/entry & /de/identifier \\
\hline /lom/general/title & /dc/title \\
\hline /lom/general/language & /dc/language \\
\hline /lom/general/description & /dc/description \\
\hline $\begin{array}{l}\text { /lom/general/keyword ou } \\
\text { /lom/classification/keyword } \\
\text { com classification/purpose igual a } \\
\text { "Discipline" ou "Idea" }\end{array}$ & $/ \mathrm{dc} /$ subject \\
\hline /lom/general/coverage & $/ \mathrm{dc} /$ coverage \\
\hline /lom/educational/learningresourcetype & /dc/type \\
\hline $\begin{array}{l}\text { /lom/lifecycle/contribute/date } \\
\text { com lifecycle/contribute/role igual a } \\
\text { "Publisher" }\end{array}$ & $/ \mathrm{dc} /$ date \\
\hline $\begin{array}{l}\text { /lom/lifecycle/contribute/entity } \\
\text { com lifecycle/contribute/role igual a } \\
\text { "Author" }\end{array}$ & $/$ dc/creator \\
\hline $\begin{array}{l}\text { /lom/lifecycle/contribute/entity } \\
\text { com o tipo de contribuição especificado } \\
\text { em lifecycle/contribute/role }\end{array}$ & /dc/othercontributor \\
\hline $\begin{array}{l}\text { /lom/lifecycle/contribute/entity } \\
\text { com lifecycle/contribute/role igual a } \\
\text { "Publisher" }\end{array}$ & /de/publisher \\
\hline /lom/technical/format & $/ \mathrm{dc} /$ format \\
\hline /lom/rights/description & /dc/rights \\
\hline /lom/relation/resource/description & /dc/relation \\
\hline $\begin{array}{l}\text { /lom/relation/resource } \\
\text { com relation/kind igual a "IsBasedOn" }\end{array}$ & $/ \mathrm{dc} /$ source \\
\hline
\end{tabular}

A partir da Tabela 1, relativa à correspondência entre SCORM e padrões de metadados Dublin Core, é possível analisar uma projeção para os resultados esperados através do trabalho de mapeamento de ontologia híbrida proposto, o que implica, por sua vez, um procedimento semelhante ao que será aplicado aos padrões de metadados SCORM e MPEG-21.

Pode-se notar a sintaxe da linguagem XPath usada para o mapeamento e de correspondência entre ambas as estruturas de metadados de representação de metadados.

Foram estabelecidos pontos de pareamento entre os 15 (quinze) elementos apresentados no padrão Dublin Core e outros 15 elementos equivalentes encontrados dentro da estrutura de metadados SCORM, que de fato corresponde à estrutura do padrão LOM, conforme já explicado neste artigo.

\section{Metadados MPEG-21 Para TV Digital E As TICE}

Em [6], [1], [7] e [8], temos amostras de que a discussão envolvendo MPEG-7, MPEG-21 e TV-Anytime vem de longa data no universo da TV Digital e, mesmo a integração entre estes já foi discutida com propostas desenvolvidas por [9] e [10], assim como a integração da TV Digital com o padrão SCORM [11].

O MPEG-21 tornou-se a plataforma modular de desenvolvimento (o framework) [12] e normalização, no sentido da integração global de todos os documentos multimídia. O multimídia não é produto de uma área do conhecimento específica, mas é uma consequência direta da normalização das práticas digitais, como os desenvolvedores de telefonia, de audiovisual, da informática.

A normalização das TIC se situa mais e mais como uma prática consistindo em oferecer plataformas de produção, ou frameworks, à indústria. Apesar do MPEG-21 ter surgido de uma comunidade que se concentra sobre o áudio e o vídeo, o chamado MPEG-21 Framework [12] pode acolher todo tipo de objetos digitais complexos, como o texto eletrônico, revistas digitais, dados científicos etc. A força deste formato reside então na sua capacidade em oferecer uma plataforma geral padronizada para o sistema de produção e distribuição de todo conteúdo digital.

Como se pode constatar a partir da literatura científica [13], [7] e [1] e da própria norma [3], o padrão MPEG-21 tem uma estrutura não rígida de metadados, e a Parte 2 da norma, DID, trata do Item Digital como a abordagem mais genérica para este propósito de uso de descrição estrutural dos metadados em objetos digitais de todo tipo.

A norma MPEG-21 comporta, hoje, 21 partes [3]. MPEG-21 é uma especificação de metadados baseada em $X M L$ que traz dois pilares fundamentais: a definição de uma unidade ou objeto fundamental de distribuição e transação, que é o Item Digital; e a noção de "leitor" (reader) - a concepção dos usuários que interagem com este.

O conceito central do MPEG-21 é o de DI - Digital Item, ou Item Digital, definido na Parte 2 da norma [12].

O DID, ou Digital Idem Declaration, é um produto digital que pode ser simples ou composto. Um exemplo típico de um DI é aquele de uma página web, contendo diferentes recursos multimídia, como texto, imagem, vídeo, elementos de formatação (como folhas de estilo CSS), hiperlinks, scripts de programação dinâmica. 
O uso do MPEG-21 DIDL (Digital Idem Declaration Language) como padrão genérico para a representação, catalogação e armazenamento de objetos digitais de aprendizagem em biblioteca já foi proposto por [14]. Demonstrou-se a aplicabilidade do DIDL para representação de objetos complexos de qualquer tipo de mídia ou conteúdo, para criação de um acervo digital em biblioteca.

Em particular, as partes 2 (Digital Item Declaration) e 3 (Digital Item Identification) permitem, respectivamente, a especificação completa e estruturada dos DI; e a sua identificação e localização.

O segundo conceito fundamental no formato MPEG-21 é o da descrição da produção e da interação com as mídias, por todos os envolvidos no processo, desde o produtor de conteúdo ao usuário final. Por isto, pode-se afirmar que o objetivo principal do MPEG-21 é definir as tecnologias necessárias para suportar o intercâmbio, acesso, consumo, comércio ou manipulação de Itens Digitais de forma eficiente [12].

Deve-se destacar também a Parte 7 do padrão, DIA (Digital Item Adaptation), que padroniza os descritores e os esquemas de descrição, permitindo a adaptação dos conteúdos dos usuários, das redes, dos terminais ou ainda do ambiente de uso. Também a REL (Rights Expression Language) e a UD (User Description) tratam de descrever metadados que criem registro sobre essa relação entre produtor, conteúdo e usuários.

\section{METODOLOGIA: ONTOLOGIA DE INTEGRAÇÃO ENTRE OS PADRÕES SCORM E MPEG-21}

Uma Ontologia SCORM MPEG-21 pode ser realizada fazendo-se sua correspondência por meio da linguagem XPath, do W3C. O emprego da linguagem XPath é direcionado à realização do estudo comparado de mapeamento entre os padrões de metadados SCORM e MPEG-21, aplicando-se esta metodologia. XPath é uma linguagem mantida pelo W3C com o objetivo primário de endereçar partes de um documento XML, também usada para testar se um código corresponde a um padrão, ou a outro código.

O MPEG-21, conforme já exposto, prevê a DIDL (Digital Item Declaration Language), porém também outros esquemas e suas linguagens, em outras das muitas partes da norma, para catalogação não só dos objetos e do fluxo da informação, caso também da DII (Digital Item Identification), e da DIA (Digital Item Adaptation); mas também de direitos autorais (MPEG-21 Parte 5, REL - Rights Expression Language), CEL (Contract Expression Language), IPMP (Intellectual Property Management and Protection); e mesmo de casos de uso (UD User Description).

Para se criar uma ontologia, faz-se uma correspondência e alinhamento entre os elementos do padrão SCORM com os do padrão MPEG-21 levando-se em conta essas distintas linguagens e a importância de cada uma para o caráter extremamente abrangente e versátil do MPEG-21 como descritor de objetos, ou itens digitais.

A orientação dos trabalhos do JTC1 SC36, como se pode acompanhar por trabalhos publicados pelo IEEE [13] e [17] baseia-se essencialmente sobre a portabilidade, a interoperabilidade e a adaptabilidade das tecnologias para a educação, o ensino e a aprendizagem. O SC36 não possui, portanto, vocação para estender os trabalhos realizados por outros comitês técnicos, como o próprio SC29, o comitê do multimídia, que trata da codificação do som, imagem, da informação multimídia e hipermídia.

Entretanto, o SC36 foi pioneiro em apontar a necessidade de sinergia do SCORM com o padrão MPEG-21, proposição todavia limitada ao tratamento de questões de direito autoral, copyright e, eventualmente, do e-commerce das TICE (Parte 5 da norma).

O conjunto dos trabalhos do MPEG-21 situa-se em perfeita continuidade com aqueles realizados anteriormente no âmbito do MPEG-7. E muitos descritores do padrão MPEG-7 fazem parte do escopo do metadata schema do MPEG-21 [12].

Os descritores e esquemas de descrição correspondentes são desenvolvidos sob a responsabilidade do grupo $\mathrm{MDS}^{9}$, cuja descrição de dados é fundada sobre a linguagem semântica de marcação XML.

As inter-relações entre padrões de metadados do MPEG e do e-Learning são esquematizadas na Tabela 2 [15] e na Tabela 3 [16]:

TABELA II.

CAMPOS ABRANGIDOS PELOS PRINCIPAIS PADRÕES DAS TICE E MPEG [15]

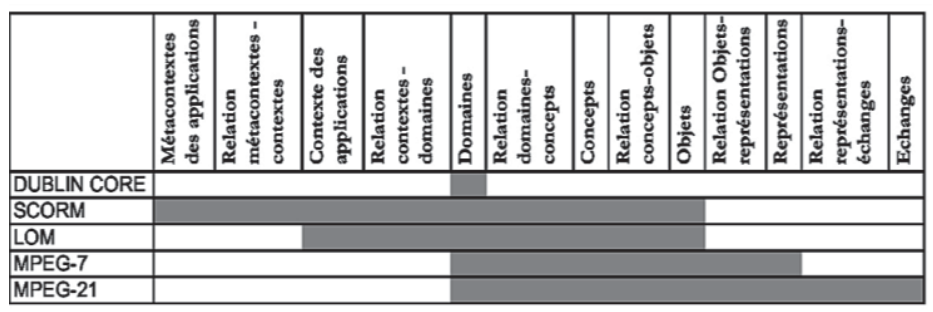

TABELA III

CAMPOS ABRANGIDOS PELOS PRINCIPAIS PADRÕES DAS TICE E MPEG [16]

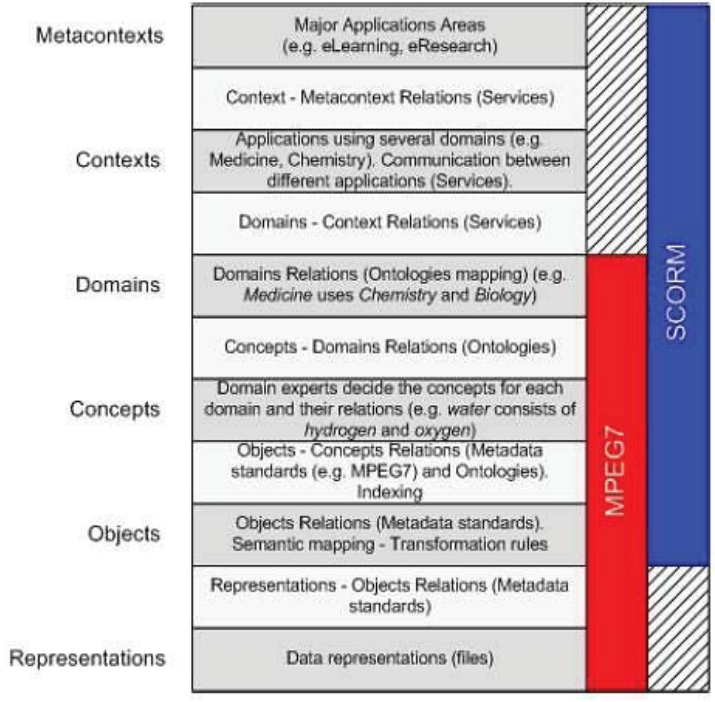

${ }^{9}$ Multimedia Desciption Schemes: grupo que trata da definição dos descritores e esquemas de descrição de metadados (metadata schema) das normas MPEG. 
Documentos DIDL são documentos XML 1.0. A sintaxe DIDL é baseada numa estrutura abstrata definida no Digital Item Declaration Model. Este modelo define os Elementos DIDL, quais sejam: Container; Item, Component, Anchor, Descriptor, Choice, Selection, Condition, Annotation, Assertion, Resource, Statement. Assim estruturalmente representados conforme as Figuras 2 e 3.

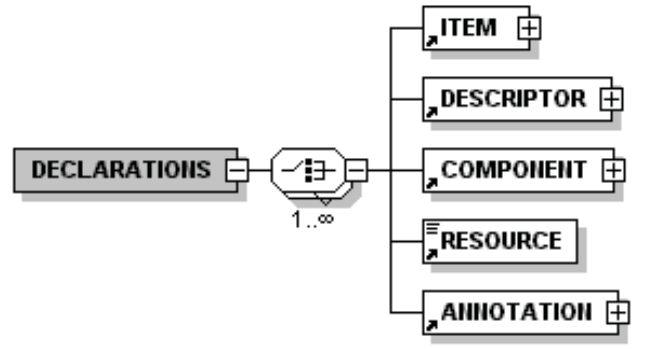

Fig. 2. Representação gráfica parcial do schema DIDL [12]. Destaque para os elementos Declarations e Item.

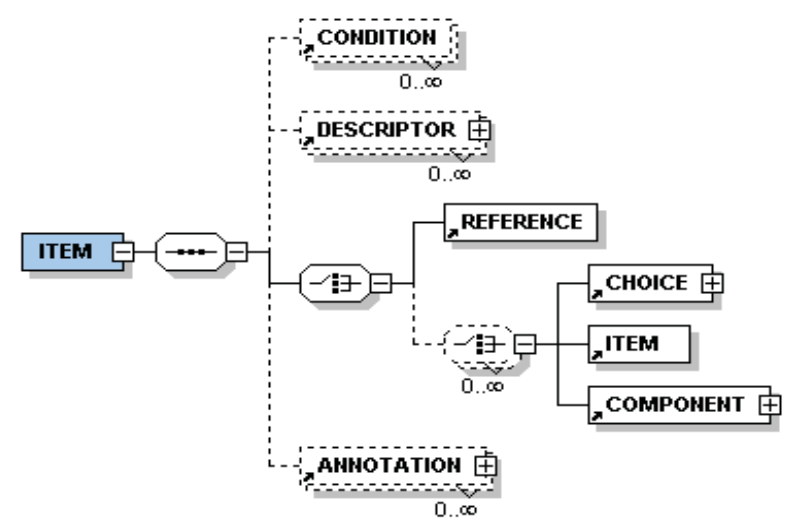

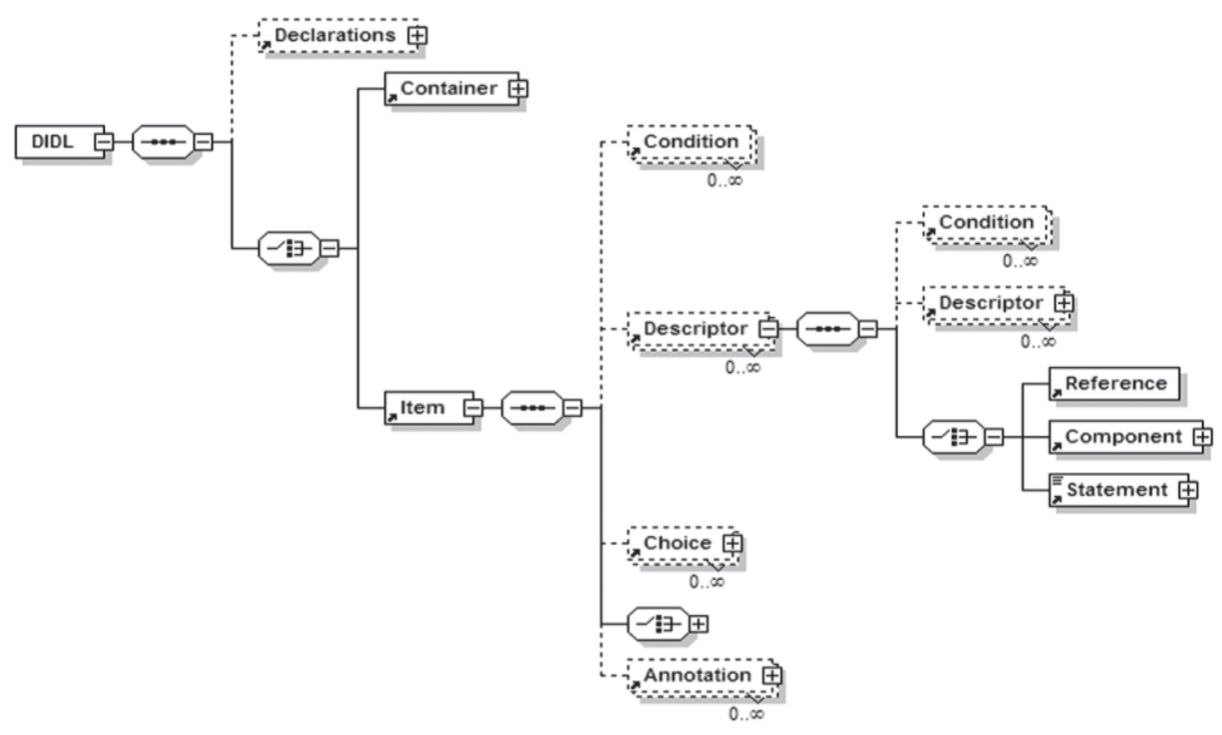

Fig. 3. Representação gráfica parcial do DIDL [17].

O código XML da DIDL relativa ao elemento Declarations (elemento especial que define uma seleção de elementos, sem instanciá-los) ficaria na seguinte forma genérica [12]:

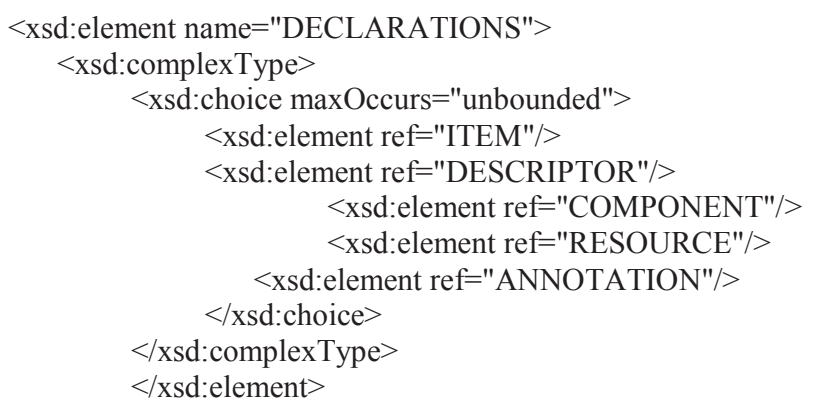




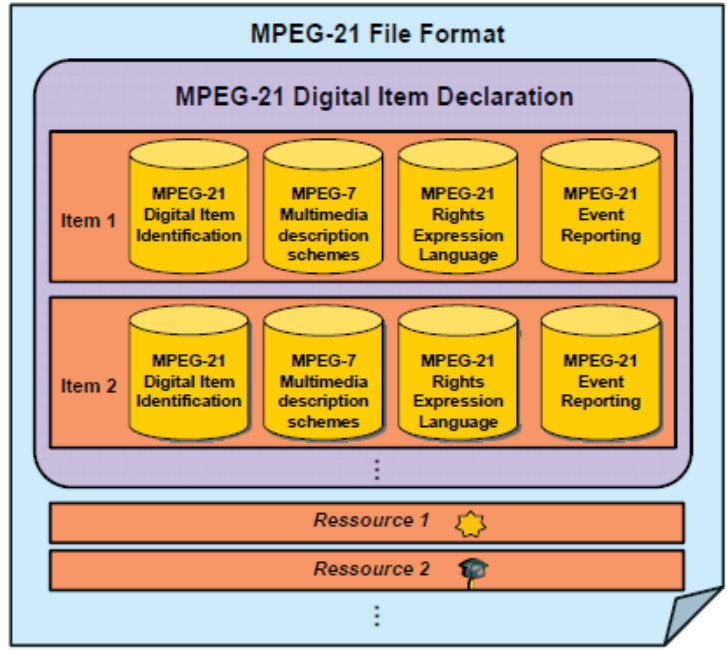

Fig. 4. Estrutura hierárquica de metadados de um arquivo MPEG-21 [18].

Para realizar-se a integração de padrões normalizados, a solução a ser implementada é fazer a correspondência entre as categorias de metadados do SCORM e os definidos pelos metadata schema do MPEG-21.

Empregando a linguagem XPath como padrão para a formatação taxonômica, inicialmente foram mapeados os seguintes metadados do padrão SCORM (LOM), que fariam correspondência ao padrão multimídia MPEG-21, aderente à convergência de plataformas. Conforme a Tabela 4:

TABELA IV

METADADOS SCORM A SEREM CORRESPONDIDOS NOS PADRÕES MULTIMÍDIA, SEJA PARA WEB, DISPOSITIVOS MÓVEIS OU TVD

\begin{tabular}{|c|c|}
\hline Metadados SCORM para Multimídia & $\begin{array}{l}\text { Metadados MPEG-21 } \\
\text { (em desenvolvimento) }\end{array}$ \\
\hline /lom/general/identifier/entry & $<$ mpeg21 $>$ \\
\hline /lom/general/title & $<$ mpeg21> \\
\hline /lom/general/language & $<$ mpeg21> \\
\hline /lom/general/description & $<$ mpeg21> \\
\hline /lom/general/keyword & $<$ mpeg21> \\
\hline /item[@identifier] & $<$ mpeg7> \\
\hline /lom/general/coverage & $<$ mpeg21 $>$ \\
\hline /lom/educational/learning/resource/type & $<$ mpeg21> \\
\hline /lom/lifecycle/contribute/role & $<$ mpeg7 $>$ \\
\hline /lom/lifecycle/contribute/date & $<$ mpeg7> \\
\hline /lom/technical/format & $<$ mpeg7 $>$ \\
\hline /lom/technical/size & $<$ mpeg7> \\
\hline /lom/technical/location & $<$ mpeg7 $>$ \\
\hline /lom/technical/duration & $<$ mpeg7> \\
\hline /lom/rights/description & $<$ mpeg21> \\
\hline /lom/relation/kind & $<$ mpeg7 $>$ \\
\hline /lom/relation/resource/description & $<$ mpeg21> \\
\hline /lom/relation/resource/catalogentry & $<$ mpeg21> \\
\hline
\end{tabular}

A Tabela 4 demonstra o mapeamento e correspondência entre os dois padrões de metadados: SCORM e MPEG- 21.

Os elementos mapeados de ambos os padrões são combinados com a finalidade de alcançar a ontologia híbrida proposta e estabelecer assim a convergência entre o padrão de metadados SCORM, para e-learning, e o padrão de metadados multimídia, o MPEG-21, ontologia aquela por característica fundamental interoperável e aplicável sobre a ampla gama de mídia digital, incluindo a web, mídias móveis ou até mesmo a televisão digital.

Esta proposta de ontologia interoperável SCORM MPEG21, empregando a linguagem Xpath, do W3C, para o seu mapeamento, tem como foco os principais itens cuja abordagem é obrigatória para se alcançar uma representação significativa dos elementos de metadados necessários para se estabelecer tanto uma descrição do item multimídia digital, quanto para se descrever objetos de aprendizagem, com o objetivo de desenvolver uma nova ontologia híbrida comum a estes dois universos de aplicação, baseada na sintaxe das preexistentes.

\section{Resultados PARCiais}

Até o presente momento conseguiu-se obter o resultado de parcial sucesso, em estágio adiantado, na execução do objetivo específico da correspondência entre padrões de metadados dos domínios do conhecimento pesquisados, que demandam integração.

O trabalho em desenvolvimento tem consistido em criar justamente uma Ontologia relativa a essas taxonomias mapeadas, a fim de se propor, a partir de tal ontologia, uma integração entre os domínios do Multimídia (inclusa a Televisão Digital) MPEG e das TICE para EaD/e-Learning.

Ou seja: a convergência entre MPEG-21 e SCORM como um padrão para descrição de objetos utilizados para catalogação e para aplicação no aprendizado eletrônico, num sentido mais amplo, e no aprendizado eletrônico via Televisão Digital numa perspectiva específica do domínio da área de concentração desta pesquisa.

Uma Ontologia SCORM MPEG-21, por meio da linguagem XPath, do W3C, já conta aqui com um desenvolvimento bem encaminhado e traz uma contribuição ao avanço do conhecimento e ao processo de normalização na área de estudo dos metadados.

Além disso, contribui a uma latente necessidade de integração entre os universos do Multimídia e das TICE, representados pelos grupos de trabalho dos comitês de padronização SC29 e SC36, da ISO/IEC JTC1, neste contexto de convergência.

\section{REFERÊNCIAS}

[1] T. Laquet, F. Prêteux, Vaucelle, A, and T. Zaharia, "Les normes MPEG7 et MPEG-21 pour la déscription des contenus multimédias," Conferência TAIMA’09, Hammamet (Tunísia), 2009.

[2] A. Vaucelle, "MPEG-21: la norme des TICE du XXIème siècle?," Rétrospective et Perspective H2PTM'09, Paris, 2009.

[3] MPEG-21 ISO/IEC N5231. MPEG21 Overview v.5. 2002.

[4] IEEE LTSC. Standard 1484.12.1 for Learning Object Metadata. New York: IEEE, 2002.

[5] ADL Advanced Distributed Learning, SCORM 2004 4th Edition Content Aggregation Model. 2009, p.4-65. 
[6] L.G.P. Alves, G. Bressan, P. Jucá. R. Kulesza, F. Silva, "Análise comparativa de metadados em TV Digital," Workshop de Televisão Digital do SBRC2006, Curitiba (Brasil), June 2006.

[7] S. Kalli, A. Lugmayr, S. Niiranen. Digital Interactive TV and Metadata: Future Broadcast Multimedia. New York: Springer, 2004.

[8] J. Hunter. Reconciling MPEG-7 and MPEG-21 Semantics Through a Common Event-aware Metadata Model. Ithaca, NY (EUA): Cornell University Library, 2002.

[9] S. Pfeiffer, U. Srinivasan, "TV-Anytime as an application scenario for MPEG-7," Proc. ACM Multimedia, Los Angeles (EUA), 2000, pp. 8992.

[10] S. Christodoulakis, F. Kazasis, P. Polydoros et al, "Ontology-Based Semantic Indexing for MPEG-7 and TV-Anytime Audiovisual Content," Multimedia Tools and Applications, vol. 26, August 2005, pp. 299-325.

[11] A. Burlamaqui et al., "T-SCORM: Uma Extensão do Padrão SCORM para Apoiar o Projeto de Conteúdos Educacionais para T-Learning," Anais do XXII SBIE - XVII WIE, Aracajú (Brasil), 2011.

[12] MPEG-21 ISO/IEC 21000-2:2003. Multimedia framework (MPEG21) Part 2: Digital Item Declaration. 2003.

[13] J. Bormans, I. Burnett, F. Pereira, R. Van Walle, K. Hill, "MPEG-21: Goals and Achievements," IEEE Multimedia, vol.10, OctoberDecember 2003, pp.60-70.

[14] J. Bekaert, P. Hochstenbach, H. Van de Sompel, "Using MPEG-21 DIDL to Represent Complex Digital Objects in the Los Alamos National Laboratory Digital Library" D-Lib Magazine, vol. 9, n. 11, November 2003.

[15] M. Ben Henda, F. Prêteux, A. Vaucelle et al. MPEG-21: une Base Normative pour le e-Procurement des TICE. Évry (França): École Télécom SudParis, 2008.

[16] L. Lyon, M. Patel, S. Christodoulakis et al., "Project n507618 DELOS: status report for work package 5. In: Joint Programme of Activities (JPA3)," February-September 2006.

[17] I. Burnett, S. Davis, G. Drury, "MPEG-21 Digital Item Declaration and Identification," IEEE Multimedia, vol. 7, n. 3, June 2005, pp.400-407.

[18] F. Schreiner. Content management and protection using Trusted Computing and MPEG-21 technologies. Dissertação (Mestrado em Informática) - Universidade Técnica de Munique (Alemanha), 2010.

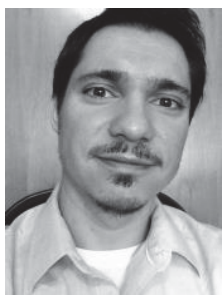

Marcelo CORREIA SANTOS Marcelo Correia Santos received his B.S. in Communication from the University of São Paulo, Brazil; MSC. degrees in Communication and Informatics Applications from the University of São Paulo State and University of Paris Sorbonne Nouvelle, in São Paulo and Paris, respectively, and is currently in process of finishing his Ph.D. followed since 2012 in Electrical Engineering - Telecommunications at Campinas State University of São Paulo. He worked as a researcher at University of São Paulo and São Paulo State from 2004 to 2011. He worked as Assistant Professor of University of São Paulo State in 2012 and as Titular Professor of SENAC University, in São Paulo, since 2013.

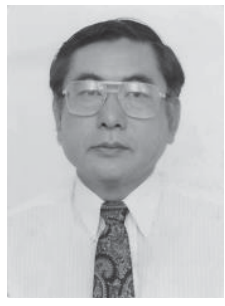

Yuzo IANO. Yuzo Iano, IEEE Member, received the B.S., M.S. and Ph.D. degrees in Eletrical Engineering from the Campinas State University of São Paulo UNICAMP in 1972, 1974 and 1986, respectively. He joined this University as a Titular Professor in 1991, where he also worked as an Assistant Professor since 1975. He served as a Telecommunications Researcher at TELEBRAS from 1986 to 1990 . He is currently the Editor in Chief of the SET International Journal of Broadcast Engineering as well. 